\title{
Adherence and Persistence Across Antidepressant Therapeutic Classes: A Retrospective Claims Analysis Among Insured US Patients with Major Depressive Disorder (MDD)
}

\author{
Katelyn R. Keyloun ${ }^{1} \cdot$ Ryan N. Hansen $^{2} \cdot$ Zsolt Hepp $^{1} \cdot$ Patrick Gillard $^{1}$ • \\ Michael E. Thase ${ }^{3} \cdot$ Emily Beth Devine $^{2}$
}

Published online: 4 April 2017

(C) The Author(s) 2017. This article is an open access publication

\begin{abstract}
Background Adherence and persistence to therapy, or how well a patient follows provider directions on frequency and time to discontinuation of prescribed medications, is associated with positive health outcomes, including decreased healthcare costs and patient mortality. A clear literature gap exists assessing adherence and persistence to antidepressants (ADs) in the major depressive disorder (MDD) population at clinically relevant time points and at the therapeutic class level.

Objective This study assessed adherence and persistence to specific ADs, therapeutic classes, and AD therapy overall at multiple time points among US individuals from commercial, Medicare supplemental, and Medicaid insurance plans.
\end{abstract}

The original version of this article was revised: The text under the heading 'Population' was incorrectly published as "38 (SD) years". The correct text should read as "38 (17) years".

A comment to this article is available at DOI 10.1007/s40263-0170419-y.

Electronic supplementary material The online version of this article (doi:10.1007/s40263-017-0417-0) contains supplementary material, which is available to authorized users.

Katelyn R. Keyloun

krkeyloun@gmail.com

1 Global Health Outcomes Strategy and Research, Allergan, 2525 Dupont Drive (T2-2P), Irvine, CA 92612-1599, USA

2 Pharmaceutical Outcomes Research and Policy Program, School of Pharmacy, University of Washington, Seattle, WA, USA

3 Perelman School of Medicine at the University of Pennsylvania, Philadelphia, PA, USA
Methods Patients with MDD without AD or MDD claims in the prior 6 months who initiated therapy in 2003-2014 with a selective serotonin reuptake inhibitor (SSRI), serotonin and norepinephrine reuptake inhibitor (SNRI), tricyclic AD (TCA), monoamine oxidase inhibitor (MAOI), or other AD were identified using MarketScan ${ }^{\circledR}$ databases. These databases contain information on diagnoses, billing codes, and dates of service. Adherence (proportion of days covered) and persistence (days until a 30-day gap in therapy) were calculated to $\mathrm{AD}$ medication, $\mathrm{AD}$ therapeutic class, and $\mathrm{AD}$ therapy overall over the first 3, 6, 9, and 12 months from the index prescription date. Multivariable logistic regression estimated the adjusted odds ratios (ORs) of adherence to initial AD medication comparing AD therapeutic classes.

Results For 527,907 patients, adherence to initial AD medication decreased over $3,6,9$, and 12 months $(41,31$, 24 , and $21 \%$, respectively). Similar patterns were observed for adherence to initial AD therapeutic class, AD therapy overall, and all three persistence calculations. The odds of adherence to SNRIs versus SSRIs were 20-27\% greater at 3, 6, 9, and 12 months (ORs 1.20, 1.23, 1.25, 1.27, respectively; $p$-values all $<0.0001$ ). Similar or significantly lower odds of adherence were demonstrated for other classes versus SSRIs at 3, 6, 9, and 12 months [ORs for other ADs $0.80,0.77,0.74,0.72$, respectively ( $p$-values all $<0.0001$ ); ORs for TCAs $0.46,0.45,0.47,0.49$, respectively ( $p$-values all $<0.0001$ ); ORs for MAOIs $1.13,1.0$, $0.77,0.69$, respectively ( $p$-values all $>0.05)]$.

Conclusion We found low adherence and persistence to ADs in the MDD population. Within the limitations of the insurance claims data we analysed, our results suggest that adherence may differ based on therapeutic class, as patients initiating SNRI therapy appeared to have a higher likelihood of adherence versus SSRIs over the year assessed, while the odds of adherence appeared similar or lower for 
other classes versus SSRIs. Further prospective research is needed to confirm these findings and determine additional drivers of these apparent differences by AD therapeutic class.

\section{Key Points}

Remaining adherent and persistent to antidepressant (AD) therapy is important to achieve therapeutic effect and potentially improve outcomes for patients with major depressive disorder (MDD).

Using data from a large US insurance claims database, we found low adherence and persistence to AD therapy among patients with MDD over the year assessed.

Results suggest that adherence and persistence may differ by therapeutic class.

\section{Introduction}

Major depressive disorder (MDD) places a large burden on public health. Recent survey data suggest an increasing trend in the prevalence of depression, both in the USA and worldwide [1, 2]. It is estimated that $6.7 \%$ of the US population aged $\geq 18$ years had a major depressive episode during 2013 [3]. In 2000, the cost of depression in the US was estimated to be $\$ 83.1$ billion of lost lifetime earnings, accounting for both direct costs of care and indirect costs due to absenteeism, presenteeism, and suicide [4].

The American Psychiatric Association (APA) supports pharmacological treatment of an episode of mild or moderate MDD with an antidepressant (AD) medication [5]. Initiation (acute)-phase treatment encompasses the first 6-12 weeks of therapy, and continuation-phase treatment is recommended for at least an additional 4-9 months. Depending on response and the severity of the presenting episode, healthcare providers may attempt to further extend therapy (maintenance phase), switch AD therapy, or combine current therapy with a second $\mathrm{AD}$ or an adjunctive agent such as an antipsychotic medication. Compliance with AD treatment guidelines is associated with decreased probability of relapse [6-8] (defined as the reoccurrence of symptoms associated with the presenting MDD episode) and increased time to relapse [9], highlighting the importance of adherence to pharmacological treatment.

Adherence, generally defined as following provider directions in regards to taking medication, is an important component of treatment [5]. Persistence is defined as the duration of time until discontinuation of medication [10]. AD medication adherence, as calculated through use of prescription claims data, is associated with positive health outcomes, such as decreased medical costs [11, 12] and potentially lower risk of mortality [13]. Adherence and persistence across AD therapy has been estimated using insurance claims data or medical chart review, yet these estimates vary across the literature and are generally limited by exclusion of at least some clinically relevant time periods (i.e., clinically relevant time periods including 3 months' acute phase, 6 months' continuation phase, and both 9 and 12 months to reflect the maximum duration of acute and continuation phases).

In 2008, Sheehan et al. [14] estimated that only $12-34 \%$ of patients with MDD remained adherent to initial AD therapy through 6 months, and, in 2010, Prukkanone et al. [15] estimated that $23 \%$ of patients were adherent through 6 months. In contrast, Wu et al. [16] estimated that $50 \%$ of patients were adherent to initial AD therapy through 6 months and Sawada et al. [17] estimated that 56\% were adherent and $44.3 \%$ were persistent to initial AD therapy through 6 months [17]. The National Center for Quality Assurance (NCQA) State of Health Care 2014 report estimated that $47-49 \%$ of patients remained on AD therapy through 6 months [18]. Taken together, these estimates represent both a large range and varied methods in calculating adherence and persistence; analyses were conducted in the USA, Japan, and Thailand using either insurance claims data or through chart review. It is also not clear whether differences in adherence or persistence across therapeutic classes exist, as few analyses have included all ADs; others have not directly compared across therapeutic classes.

Since publication of the article by Sheehan et al. [14] in 2008, rates of adherence and persistence to more recently introduced ADs (vortioxetine, levomilnacipran, vilazodone, trazodone extended release, desvenlafaxine, and selegiline transdermal patches) have not been estimated. A comprehensive analysis of adherence and persistence to AD therapy is particularly warranted in real-world settings, focusing on any differences in adherence by initial therapeutic class. Thus, the objectives of this study were to describe the MDD population through a large US insurance claims database and to calculate adherence and persistence to initial AD therapy during clinically relevant time periods and by therapeutic class, including new ADs and formulations.

\section{Methods}

\subsection{Data Source}

Truven Health Analytics MarketScan ${ }^{\circledR}$ Research Databases were used for this study to assess US individuals from 
commercial, Medicare supplemental, and Medicaid insurance plans. These databases contain inpatient and outpatient medical claims linked to prescription medication claims representing US patients from all three of these types of insurance plans. The Medicaid database lacked patient-specific region data. This research did not meet the definition of Human Subjects Research because the dataset is de-identified at its source and is compliant with the Health Insurance Portability and Accountability Act of $1996[19,20]$.

\subsection{Sample Selection}

\subsubsection{Inclusion Criteria}

Commercial, Medicare supplemental, and Medicaid databases were queried to identify claims between 1 July 2003 and 1 January 2014 (Fig. 1). Qualifying diagnoses for MDD included either one inpatient claim or one outpatient service claim with a second confirmatory claim (either inpatient or outpatient) within 60 days after the initial outpatient claim. MDD claims were identified using the International Classification of Diseases, Ninth Revision, Clinical Modification (ICD-9-CM) codes for MDD single episode (296.2), MDD recurrent episode (296.3), dysthymic disorder (300.4), and depressive disorder not otherwise specified (311) [14, 21]. The first qualifying MDD claim to occur was defined as the index diagnosis date (IDD). Patients were required to have had continuous pharmacy and medical benefit eligibility from 6 months before to 12 months after the IDD. The index prescription date (IPD) was defined as the first pharmacy claim for an $\mathrm{AD}$ medication filled within 60 days before or after a qualifying diagnosis of MDD, which is similar to a prior claims analysis [14]. Continuous insurance eligibility was re-assessed from 6 months before the IPD if the IPD preceded the IDD and 12 months after the IPD if the IPD followed the IDD. Pharmacy claims included all ADs used for the treatment of MDD (Appendix 1).

\subsubsection{Exclusion Criteria}

Patients were excluded if they had a diagnosis for MDD or an $\mathrm{AD}$ prescription claim in the 6 months before the IDD, excluding the 60-day period that preceded the IDD to account for the IPD definition. Patients who did not have continuous insurance coverage from 6 months before to 12 months after the IPD were excluded from the study. To ensure ADs were being used to treat a new diagnosis of MDD, patients with claims for certain conditions were excluded from the dataset if they occurred within 6 months before to 12 months after the IDD (Appendix 2). These include schizophrenic disorder (ICD-9 code:

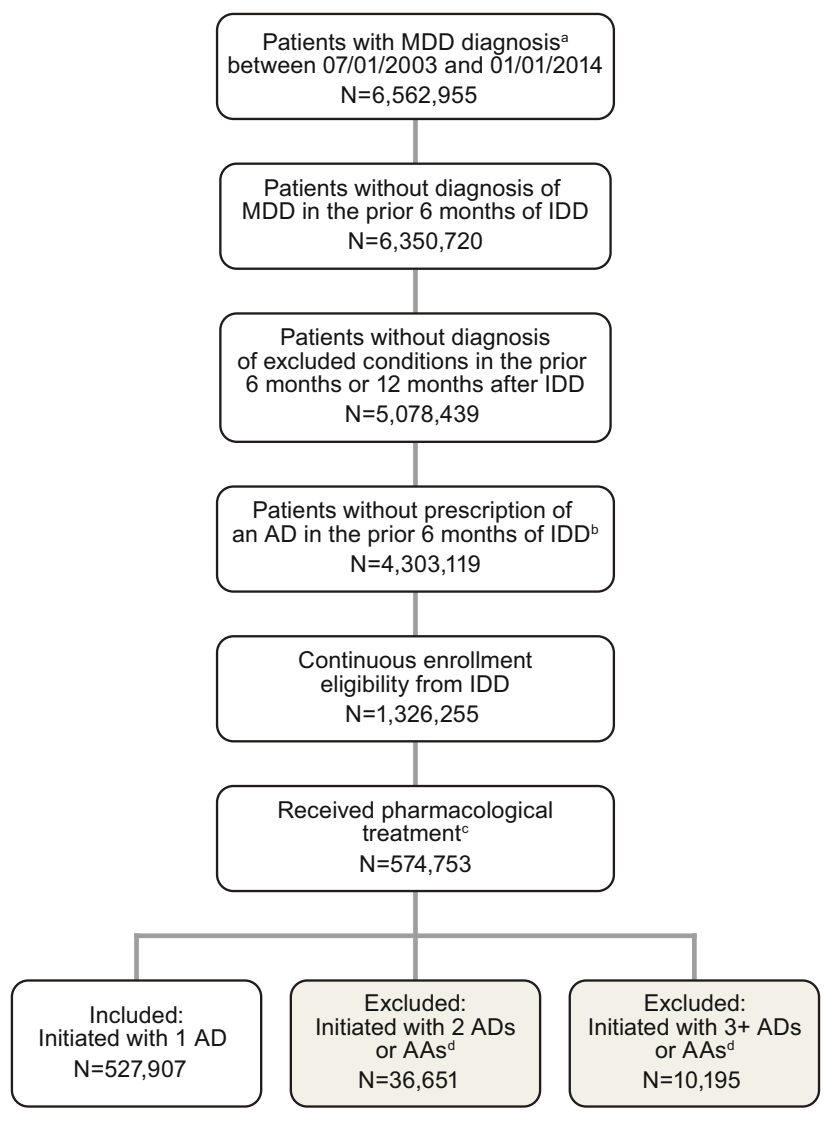

Fig. 1 Patient selection flowchart. ${ }^{a}$ One inpatient or one outpatient diagnosis with a confirmatory outpatient or inpatient diagnosis within 60 days. ${ }^{\mathrm{b}}$ This 6 -month period did not include the 60-day period immediately preceding the IDD. ${ }^{\mathrm{c}}$ If the IPD was before the IDD, continuous enrollment eligibility was re-evaluated for 6 months prior to the IPD. If the IPD was after the IDD, eligibility was re-evaluated for 12 months post IPD, as defined in the exclusion criteria. ${ }^{\mathrm{d}}$ As defined in exclusion criteria. $A A$ adjunctive agent, $A D$ antidepressant, $I D D$ index diagnosis date, IPD index prescription date, $M D D$ major depressive disorder

295), bipolar disorder (296.0-296.1 and 296.4-296.9), other psychosis-related disorders, paranoid states (297), other mood disorders (293.83 and 301.13), drug-induced depression (292.84), depressive-type psychosis (298), Alzheimer's disease (331), Parkinson's disease (332), and dementia (290) [12, 14, 22]. In addition, patients who had a claim for pregnancy (630-679) were excluded from the dataset because of the effect pregnancy may have on discontinuation of therapy. Patients were excluded if they initiated with two medications or had more than three medications prescribed within the first 60 days after the IPD. Initiating with two medications was defined as having two prescriptions for AD therapy on the IPD (or one $\mathrm{AD}$ and one adjunctive agent) or prescriptions for two $\mathrm{ADs}$ (or one $\mathrm{AD}$ and one adjunctive agent) that overlapped for more than 30 days within the first 60 days of the IPD (Appendix 1; therapeutic classifications were 
based on US Pharmacopeial Convention Medicare Model Guidelines v6.0 [23]). Overlapped days were not required to be consecutive. If patients did not meet the definition of initiating with two medications or more than three medications as defined, no further exclusion criteria were applied.

\subsection{Calculation of Adherence and Persistence}

Adherence was calculated considering adherence to the initial $\mathrm{AD}$ medication, to the initial $\mathrm{AD}$ therapeutic class, and to AD therapy overall (Fig. 2). Monotherapy was assumed for calculations of adherence. Each calculation was assessed at 3, 6, 9, and 12 months after the IPD. The 3-month time frame was chosen based on the Antidepressant Medication Management (AMM) quality measure for effective initiation treatment [24]. The 6-month time frame was chosen based on the minimum duration of therapy to achieve both initiation and continuation treatment, its use in the AMM quality measure for effective continuation treatment [5], and use in prior adherence and persistence analyses $[14-17,25,26]$. Adherence was also calculated at 9 and 12 months, as the added duration of initiation and continuation phase allows up to a full year of therapy $[5,27]$. Calculations of adherence such as medication possession ratio (MPR) and proportion of days covered
(PDC) are well-defined [10, 12, 28, 29]. PDC was chosen as the primary calculation of adherence because it accounts for overlap of prescriptions and is considered a preferred measure of adherence [30]. MPR was also calculated to ensure similar results between calculations. MPR values over 1.0 were truncated to 1.0. Both PDC and MPR were calculated over each time frame as the total number of days that the medication was available or the days' supply, respectively, and divided by the period of follow-up. Adherence was dichotomized as adherent if PDC (or MPR) was $\geq 0.80$ and not adherent if PDC (or MPR) was $<0.80$, as this threshold is commonly used and supported to have predictive ability to estimate risk of hospitalization [31].

Persistence was also calculated considering persistence to the initial AD medication, to the initial therapeutic class, and to AD therapy overall. Each calculation was also assessed at 3, 6, 9, and 12 months after the IPD. Persistence was defined as the days of treatment until a 30-day gap in therapy, and the gap was chosen based on the AMM quality measure for effective acute-phase treatment [24] and use in prior claims analyses [32,33]. Persistence was calculated until patients discontinued therapy, were lost to follow-up due to loss of insurance eligibility after a minimum of 12 months' continuous enrollment, or were followed for a maximum of 2 years ( 730 days), whichever occurred first. Persistence in days was similarly converted
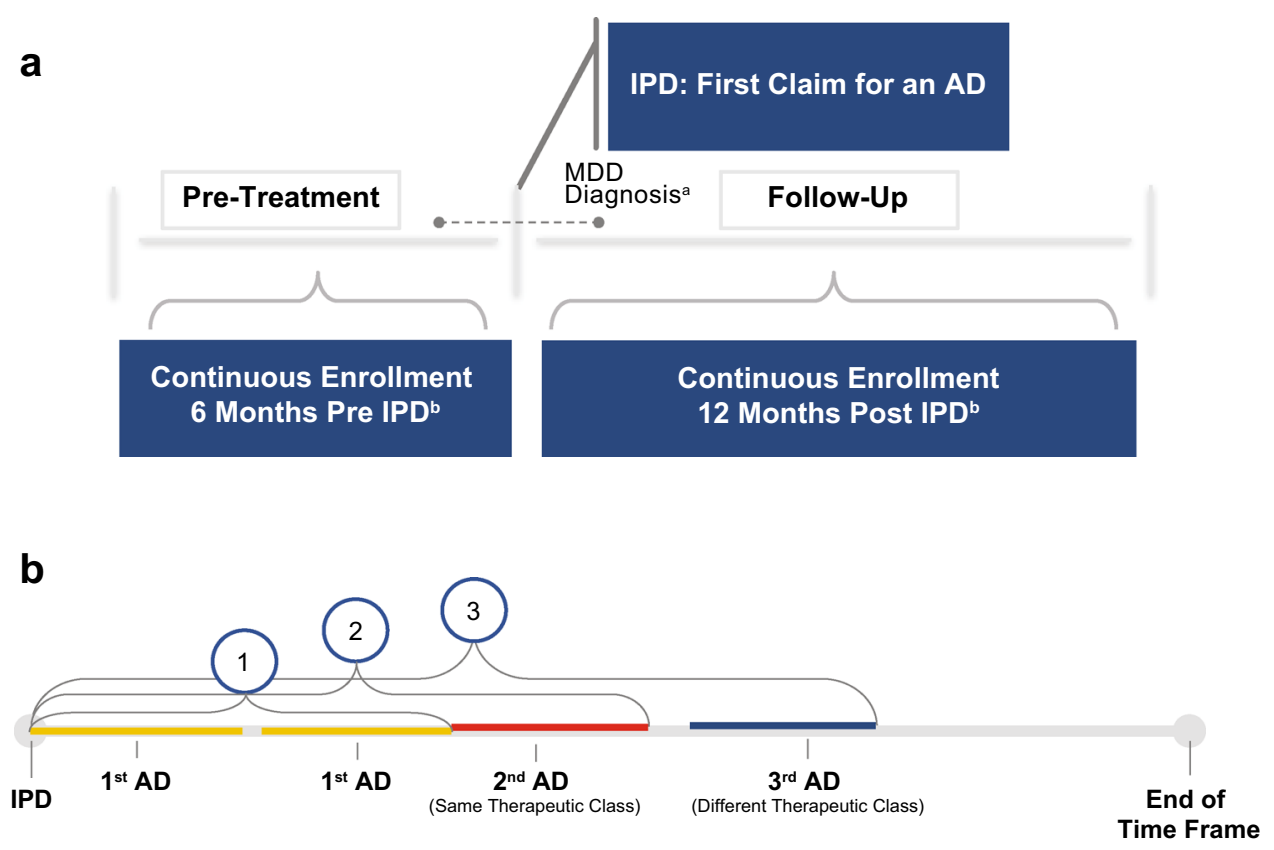

Fig. 2 Index prescription date identification (a) and identification of relevant prescription claims to calculate adherence and persistence (b). ${ }^{\mathrm{a}} \mathrm{IPD}$ was the first claim for an $\mathrm{AD}$ prescription that occurred within 60 days before or after a qualifying MDD diagnosis date. ${ }^{\mathrm{b}}$ Continuous enrollment assessed principally from the IDD and was then re-assessed from the IPD. b Consider a patient who receives citalopram (first and second prescription; yellow), escitalopram (third prescription; $r e d$ ), and then desvenlafaxine (fourth prescription; blue). Prescription claims relevant to calculations are identified by calculation type: (1) to initial $\mathrm{AD}$ medication (yellow prescription claims only), (2) to initial therapeutic class (yellow and red prescription claims only), and (3) to $\mathrm{AD}$ therapy overall (yellow, red, and blue prescription claims). $A D$ antidepressant, $I D D$ index diagnosis date, $I P D$ index prescription date, $M D D$ major depressive disorder 
to a dichotomous outcome as persistent or not persistent to each time frame.

\subsection{Demographic and Clinical Variables}

Baseline demographic covariates included age, sex, source of insurance claim, region in which medical care was received, and type of health plan. Clinical covariates included MDD diagnosis code (single episode, recurrent episode, dysthymic disorder, depression not otherwise specified) and Charlson Comorbidity Index (CCI) score ( 0 , 1, 2, 3, or greater). The CCI was calculated as an indicator of mortality risk over the year prior to the IDD using the Deyo modification [34].

\subsection{Subgroups of Interest}

Patients with a diagnosis of anxiety and chronic non-cancer pain disorders were of interest because certain AD therapy is US FDA labeled or used off-label to treat these comorbidities. Diagnosis of cancer pain was not of interest because guidelines support analgesic therapy for management of pain [35]. Patients were identified through ICD-9 codes [14, 26, 36, 37] (Appendix 2) within 60 days before or after the IDD. For the chronic non-cancer pain disorder of chronic low back pain, claims were identified up to 6 months before the IDD or 60 days after, to account for treatment progression over time.

\subsection{Statistical Analyses}

Descriptive statistics were used to characterize baseline demographics, clinical characteristics, and subgroups. Means and standard deviations (SDs) were used to describe continuous variables, and counts and proportions were used for categorical variables. Bivariate statistics for differences in characteristics across therapeutic classes were conducted using Chi squared $\left(\chi^{2}\right)$ tests for categorical variables and analysis of variance (ANOVA) for continuous variables. Adherence outcomes and persistence outcomes were interpreted based on descriptive results and compared using inferential statistics. Specifically, we compared the proportion adherent or persistent from the IPD to 3-month time frame separately to each other timeframe (IPD to 6, 9, and 12-months time frames) using McNemar's $\chi^{2}$ tests. Persistence on therapy (time to discontinuation) between therapeutic classes was compared using the Kaplan-Meier estimator and log-rank test.

Unadjusted logistic regressions were conducted on the complete cohort to estimate the odds of adherence to initial therapeutic class versus selective serotonin reuptake inhibitor (SSRI) therapy for each time frame (from IPD to 3, 6,
9, or 12 months). The SSRI referent group was chosen based on a majority of the cohort having been initially prescribed SSRI therapy. Multivariable logistic regression was conducted to provide adjusted estimates for each time frame, and the model included all baseline demographic and clinical characteristics as well as the presence of a comorbid anxiety or chronic non-cancer pain disorder. Covariates for the adjusted model were chosen based on statistical, clinical, and epidemiological rationale. All analyses were performed using the statistical software package STATA ${ }^{\circledR}$ version 13 [38]. A significance level of $\alpha=0.05$ was chosen for all analyses.

\subsection{Sensitivity Analyses}

A sensitivity analysis was performed for persistence using a 45-day gap in therapy as a cut-off and was compared with the primary analysis that used a 30-day gap in therapy as a cut-off.

\section{Results}

\subsection{Population}

Of the estimated 200 million patients in Marketscan ${ }^{\circledR}$ Databases, we identified 6,562,955 patients with qualifying diagnoses of MDD from 1 July 2003 to 1 January 2014 (Fig. 1). After inclusion and exclusion criteria were applied, 574,753 patients received a new prescription for AD therapy. Of the 574,753 patients, another 36,651 were excluded because they initiated with two ADs and augmentative agents and another 10,195 because they initiated with three or more ADs and augmentative agents. Finally, 527,907 patients were included in the analysis of adherence and persistence. The left-skewed distribution of initial prescriptions indicated higher prescription density during the later years of the selection period, yet this increase in density was similar across therapeutic classes (Fig. 3).

The average (SD) age of the study population was 38 (17) years. The majority of subjects were female (64\%) and covered by commercial insurance plans $(81 \%)$; preferred provider organizations and health maintenance organizations (48 and 20\%) were the most common insurance plan types (Table 1; Appendix 3). The largest proportion of the sample resided in the southern region of the USA (29\%). Of the four MDD-specific diagnostic codes, the most common index diagnosis was depression not otherwise specified (56\%). The analytic cohort had low mortality risk, as demonstrated by the majority $(79 \%)$ having a CCI score of 0 , yet $24 \%$ had comorbid anxiety disorders, $24 \%$ had comorbid chronic non-cancer pain 
Fig. 3 Distribution of index prescriptions by year and initial therapeutic class. $A D$ antidepressant, $M A O I$ monoamine oxidase inhibitor, SNRI serotonin and norepinephrine reuptake inhibitor, SSRI selective serotonin reuptake inhibitor, $T C A$ tricyclic antidepressant

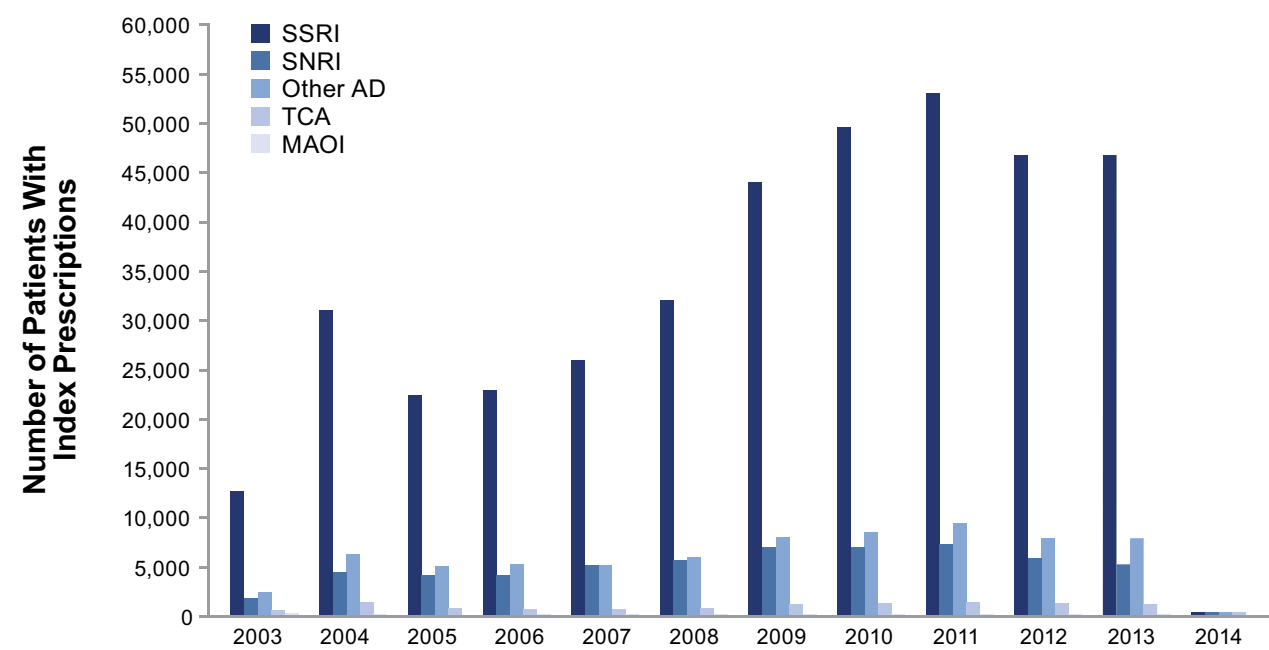

disorders, and $6 \%$ had both. The proportion of patients with comorbid anxiety disorders was similar across therapeutic classes, whereas a greater proportion of patients with comorbid chronic non-cancer pain disorders were initially prescribed serotonin and norepinephrine reuptake inhibitors (SNRIs) and tricyclic ADs (TCAs) in comparison with other therapeutic classes (31 and 52\%, respectively). The most commonly prescribed AD therapeutic class was SSRI (73.5\% of cohort). The most common SSRI prescribed was sertraline $(18.7 \%$ of cohort), and the most common AD prescribed from the other $\mathrm{AD}$ therapeutic classes was bupropion extended release (5.4\%). No patients received an initial isocarboxazid prescription, probably because it is used as a second-line therapy, or initial prescriptions for vortioxetine or levomilnacipran, most likely because they were only recently approved by the FDA to treat MDD $[39,40]$.

\subsection{Adherence and Persistence}

Both adherence and persistence decreased significantly over the year of therapy for each of the three calculations: to initial $\mathrm{AD}$ medication, initial therapeutic class, and $\mathrm{AD}$ therapy overall. Yet, adherence and persistence were slightly higher to initial therapeutic class and to AD therapy overall when compared with initial AD medication. Adherence at 6 months by the three calculations was 31, 33 , and $35 \%$ (Fig. 4a), where persistence at 6 months was 31,33 , and $36 \%$ (Fig. 4b). When adherence and persistence to initial $\mathrm{AD}$ medication were stratified by therapeutic class, both similarly decreased over the year of therapy (Fig. 5a, b). Patients initially prescribed SNRIs had the highest adherence and persistence at all time frames (at 6 months, 37 and 37\%, respectively) compared with the other therapeutic classes, whereas TCAs had the lowest adherence and persistence (at 6 months, 16 and 17\%, respectively). Adherence was slightly higher when calculated using MPR, yet the results were similar.

Upon sensitivity analysis, persistence was slightly higher using the 45-day persistence cut-off versus the 30-day cut-off, yet the results were similar across therapeutic classes over the year of therapy. Persistence at 6 months using a 45 -day gap was $37 \%$ for SSRIs, $30 \%$ for other ADs, $42 \%$ for SNRIs, $20 \%$ for TCAs, and $41 \%$ for monoamine oxidase inhibitors (MAOIs).

A Kaplan-Meier time-to-discontinuation curve of initial AD medication, by therapeutic class, described the persistence outcome continuously (Fig. 6). The commonality among insurance plans of covering a 30-day supply of medication at a time likely accounted for the pattern of drop-off at 30,60, and 90 days. The curves differed by therapeutic class ( $p$-value $<0.0001$ ), with lower persistence to initial TCA therapy at all time points, and at 270 days, with lower persistence to initial MAOI therapy.

Our logistic regression results found differences in the odds or likelihood of adherence to initial AD medication across therapeutic classes. The adjusted odds of adherence at 6 months were $23 \%$ higher for patients initiating with SNRI therapy versus SSRI therapy [Fig. 7; odds ratio (OR) 1.23 ; 95\% confidence interval (CI) 1.21-1.25; pvalue $<0.0001]$. For patients initiating with the other $A D$ or TCA therapeutic classes, the adjusted odds of adherence at 6 months were $23 \%$ (OR 0.77 ; 95\% CI $0.75-0.78$ ) and $55 \%$ (OR $0.45 ; 95 \%$ CI $0.42-0.47$ ) lower, respectively ( $p$-values $<0.0001$; Fig. 7). Adherence among patients initiating with MAOIs was not significantly different compared with those initiating with SSRIs. Similar results were found to the 3-, 9-, and 12-months time frames (Appendix 4). 
Table 1 Demographic and clinical characteristics of the cohort and presence of anxiety or chronic pain disorders

\begin{tabular}{|c|c|}
\hline Characteristics & $\begin{array}{l}\text { All antidepressant classes } \\
(N=527,907)\end{array}$ \\
\hline \multicolumn{2}{|l|}{ Demographic variables } \\
\hline \multicolumn{2}{|c|}{ Antidepressant class [no. of prescriptions (\%)] } \\
\hline SSRI & $387,746(73)$ \\
\hline Other AD & $71,664(14)$ \\
\hline SNRI & $57,342(11)$ \\
\hline TCA & $11,070(2)$ \\
\hline MAOI & $85(<1)$ \\
\hline Mean age (SD), years & $38(17)$ \\
\hline \multicolumn{2}{|l|}{ Age categories, years } \\
\hline$<18$ & $83,293(16)$ \\
\hline $18-39$ & $192,724(37)$ \\
\hline $40-65$ & $222,532(42)$ \\
\hline$>65$ & $29,358(6)$ \\
\hline \multicolumn{2}{|l|}{ Sex } \\
\hline Female & $338,226(64)$ \\
\hline Male & $189,681(36)$ \\
\hline \multicolumn{2}{|l|}{ Insurance claim source } \\
\hline Commercial & $425,106(81)$ \\
\hline Medicaid & $71,710(14)$ \\
\hline Medicare & $31,091(6)$ \\
\hline \multicolumn{2}{|l|}{ Geographical location } \\
\hline South & $150,913(29)$ \\
\hline Northcentral & $127,340(24)$ \\
\hline West & $99,265(19)$ \\
\hline Northeast & $72,143(14)$ \\
\hline Missing & $71,710(14)$ \\
\hline Unknown & $6536(1)$ \\
\hline \multicolumn{2}{|l|}{ Health plan type } \\
\hline PPO & $255,337(48)$ \\
\hline HMO & $103,521(20)$ \\
\hline Comprehensive & $75,235(14)$ \\
\hline POS & $38,915(7)$ \\
\hline CDHP & $16,164(3)$ \\
\hline Missing & $15,282(3)$ \\
\hline POS with capitation & $10,772(2)$ \\
\hline HDHP & $8208(2)$ \\
\hline EPO & $4473(<1)$ \\
\hline \multicolumn{2}{|l|}{ Clinical variables } \\
\hline \multicolumn{2}{|l|}{ MDD diagnosis code } \\
\hline Depression, NOS & $293,311(56)$ \\
\hline Recurrent episode & $95,652(18)$ \\
\hline Single episode & $71,942(14)$ \\
\hline Dysthymia & $67,002(13)$ \\
\hline \multicolumn{2}{|c|}{ CCI (Deyo Modification) in the 12 months pre-IDD } \\
\hline 0 & $419,089(79)$ \\
\hline 1 & $70,158(13)$ \\
\hline 2 & $19,370(4)$ \\
\hline
\end{tabular}

Table 1 continued

\begin{tabular}{cc}
\hline Characteristics & $\begin{array}{l}\text { All antidepressant classes } \\
(N=527,907)\end{array}$ \\
\hline $3+$ & $19,290(4)$ \\
$\begin{array}{c}\text { Subgroups of interest } \\
\text { Comorbid diagnoses }\end{array}$ & \\
Anxiety disorder & $125,319(24)$ \\
Chronic non-cancer pain disorder & $124,170(24)$ \\
\hline
\end{tabular}

Data are presented as $n(\%)$ unless otherwise indicated

The CCI was calculated using the Deyo modification in the year prior to the index MDD diagnosis date

$A D$ antidepressant, $C C I$ Charlson Comorbidity Index, $C D H P$ consumer-driven health plan, EPO exclusive provider organization, $H D H P$ high deductible health plan, $H M O$ health maintenance organization, $I D D$ index diagnosis date, $M A O I$ monoamine oxidase inhibitor, $M D D$ major depressive disorder, NOS not otherwise specified, $P O S$ point of service, $P P O$ preferred provider organization, $S D$ standard deviation, SNRI serotonin and norepinephrine reuptake inhibitor, SSRI selective serotonin reuptake inhibitor, TCA tricyclic antidepressant

${ }^{a}$ Comorbid anxiety disorders and chronic non-cancer pain disorder were identified within 60 days before or after the IDD, with the exception of the chronic non-cancer pain disorder: low back pain (Appendix 2). For this disorder only, chronic low back pain was identified up to 6 months before the IDD to 60 days after to capture the progression of treatment over time

\section{Discussion}

The purpose of this analysis was to assess and compare adherence and persistence to initial AD medication across therapeutic classes for the MDD population. It is the largest retrospective study using insurance claims data to date for an MDD-specific population, in both sample size and number of ADs included. The results support that adherence and persistence is low across therapeutic classes in a "real-world" setting. Similar to previously reported estimates, this analysis found that the proportion adherent at 6 months was higher with SNRI or SSRI therapy than with TCAs $[14,41]$. Yet, the proportion adherent at 6 months to MAOIs was similar to SSRI or SNRI therapy, which was unexpected and likely due to the small sample size $(n=85)$. The proportion persistent was also consistent with the literature [17, 26, 42].

Adherence and persistence calculations were compared to the initial $\mathrm{AD}$ medication, to initial therapeutic class, and to AD therapy overall. Adherence to initial class and overall to AD therapy was slightly higher than adherence to initial AD medication, indicating that a small subset of patients who discontinue their initial AD medication switch to another $\mathrm{AD}$ from the same or a different therapeutic class. However, for the majority of patients, AD therapy was discontinued much earlier than the clinically recommended 6- to 9-months period, and this discontinuation 
Fig. 4 Adherence (a) and persistence (b) by calculation type over the first $3,6,9$, and 12 months after index prescription date. $A D$ antidepressant. $* p<0.0001$ vs. adherence to 3 months (McNemar's paired $\chi^{2}$ test of equality of proportions)

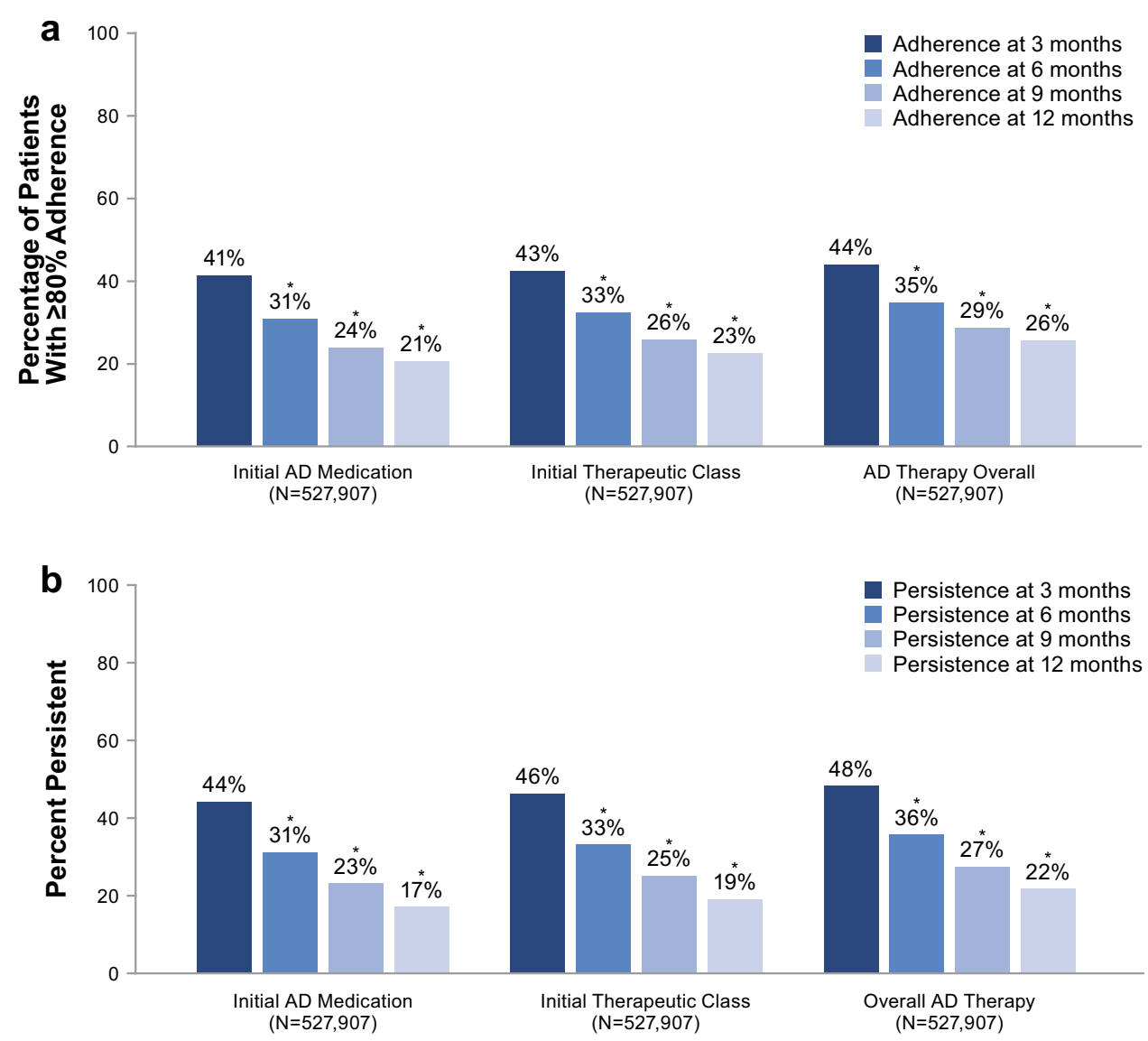

primarily occurred over the first 3 months of therapy. Results suggest that switching between ADs did not fully account for low adherence and persistence over the year of therapy assessed. Our findings support that adherence and persistence to AD therapy is similar to or lower than that for the suboptimal medication adherence and persistence reported for other chronic disease states, including hypertension, gout, overactive bladder syndrome, glaucoma (to prostaglandin eye-drop therapy), and diabetes (to oral glycemic control medications) [43, 44]. This demonstrates suboptimal adherence and persistence to AD therapy in the MDD population.

The likelihood of remaining adherent over the year of therapy differed by the initial therapeutic class prescribed, highlighting the potential importance of initial choice of AD therapy. Those initially prescribed SNRI therapy had increased odds of adherence versus those receiving SSRI therapy over the year of therapy, whereas those initially prescribed ADs from the other AD classes and TCA class had lower odds of adherence than those receiving SSRIs. The odds of adherence for patients initially prescribed MAOIs were no different from those receiving SSRI therapy. Interestingly, the odds of adherence differed by MDD diagnosis code. Specifically, patients with an index diagnosis of dysthymic disorder had increased odds of adherence to initial therapy compared with patients diagnosed with a single episode of MDD, after adjusting for all other covariates (Appendix 4).

Adherence may be related to a perceived or experienced clinical benefit to the patient. A review conducted in 2005 suggested a potential difference in the rate of response to therapy and remission with venlafaxine versus other antidepressants [45]. Although evidence suggests adverse events may be similar when comparing SNRIs and SSRIs, SNRIs may pose an advantage to treating depression-associated neuropathic pain and other pain.

\section{Limitations}

Use of insurance claims data may pose a risk of misclassification bias because of entry errors and consolidation of information across health plans, or incomplete information, as insurance claims that are not linked with an individual's primary insurance may not be captured. Importantly, clinical and demographic information in claims lack validation to medical record data. The prevalence of $\mathrm{AD}$ prescription in the sample also may not reflect prescribing practices, as patients may not fill $\mathrm{AD}$ prescriptions that they receive from healthcare providers. Yet, as our sample was large 

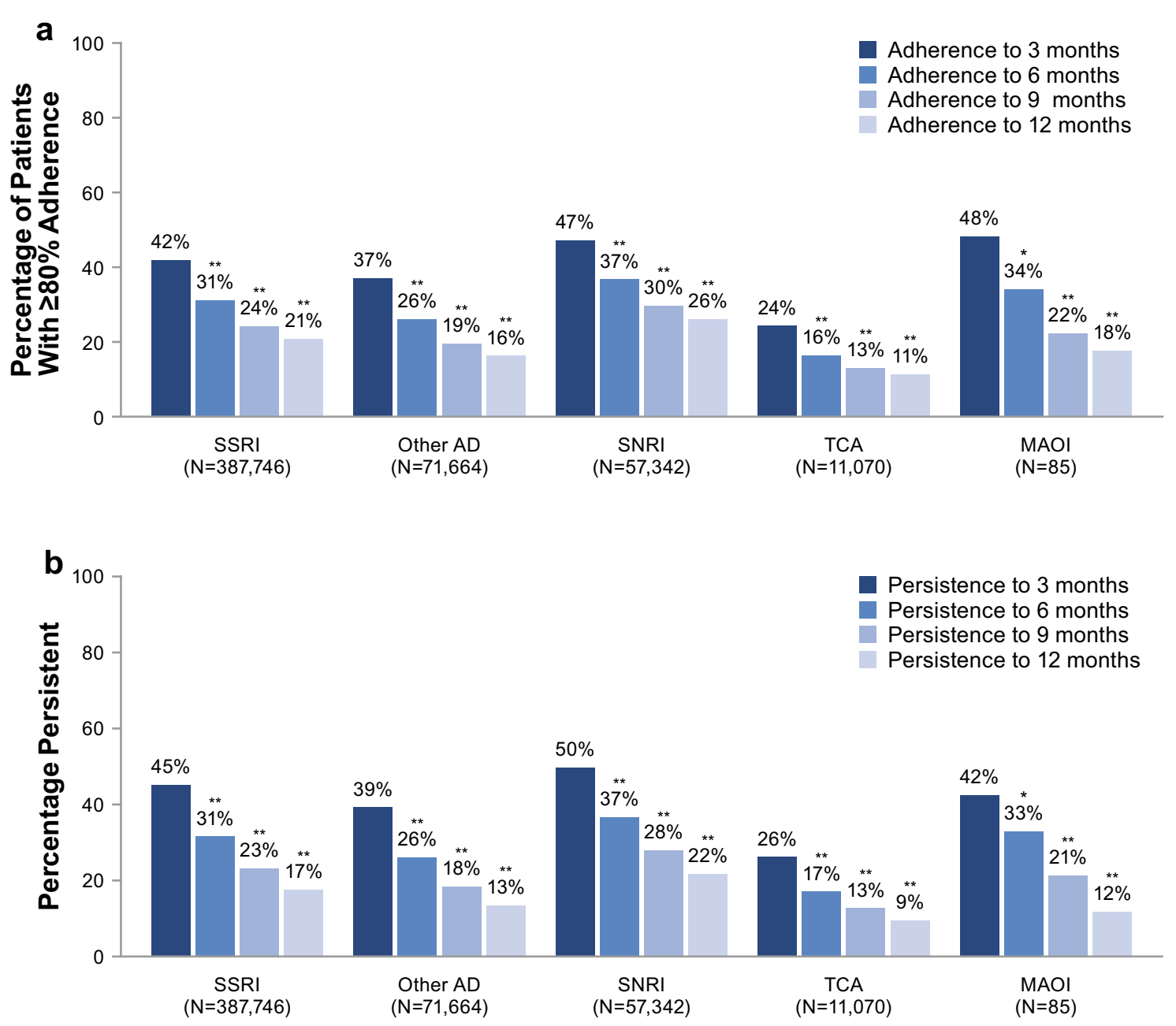

Fig. 5 Proportion adherent (a) and persistent (b) to initial antidepressant medication at the first 3, 6, 9, and 12 months after index prescription date, by therapeutic class. $A D$ antidepressant, MAOI monoamine oxidase inhibitor, SNRI serotonin and norepinephrine

and diverse, prescribing patterns likely echoed current practice. Assessing adherence and persistence through analyses of claims data may not reflect true adherence and persistence, as our calculations assume patients take the prescriptions they fill. Alternative clinical instructions or use may also lead to underestimation of adherence. For example, the SSRI trazodone is commonly used off-label at low doses for its sedative properties and is not typically used to treat MDD [5], and new clinical guidance for elderly patients with MDD may contribute to discontinuation of therapy per provider instruction.

While observational studies such as ours are necessary for evaluating "real-world" conditions, they have a higher potential for bias than randomized controlled trials. Use of claims data did not allow for control of all possible factors that may be associated with adherence, including clinical factors related to patient care. To the extent possible, we addressed this by including many potential confounders and precision reuptake inhibitor, $S S R I$ selective serotonin reuptake inhibitor, $T C A$ tricyclic antidepressant. $* p<0.01, * * p<0.0001$ vs. adherence to 3 months (McNemar's paired $\chi^{2}$ test of equality of proportions)

variables in the adjusted analyses: age, sex, source of insurance, geographical region, insurance plan type, MDD diagnosis code, CCI, and presence of anxiety or chronic noncancer pain comorbidities. Multivariable logistic regression was used to adjust for these variables; although a method applying propensity score matching was considered, results were expected to be similar because the same covariates would be included to create the propensity score. Certain clinical and psychosocial factors that may be associated with adherence, such as the provider's title (e.g. family practitioner vs. psychiatrist), provider practice models, past treatment for MDD, clinical response and occurrence of adverse drug events, patient beliefs, patient-provider relationship, patient education, and patient economic and cultural factors, were not available in the insurance claims database [5].

Our study strengthens the need for future research in another database or with prospectively collected data that characterizes clinical and psychosocial factors that may 


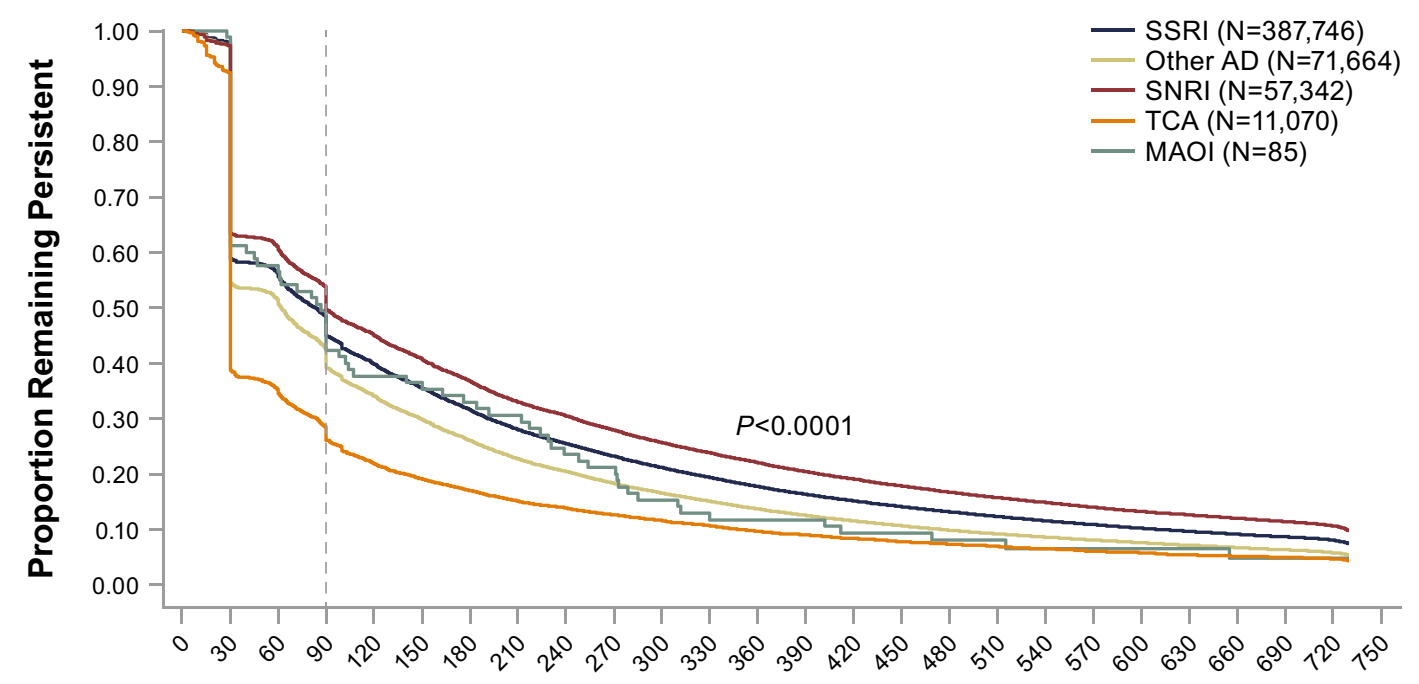

Days to Discontinuation

Fig. 6 Time to discontinuation of initial antidepressant medication by therapeutic class. $A D$ antidepressant, $M A O I$ monoamine oxidase inhibitor, SNRI serotonin and norepinephrine reuptake inhibitor, SSRI selective serotonin reuptake inhibitor, TCA tricyclic antidepressant. $p<0.0001$ comparing therapeutic classes (log-rank test)

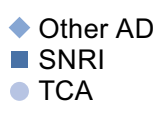

SNRI

TCA
$1+4$

$(0.75-0.78)$

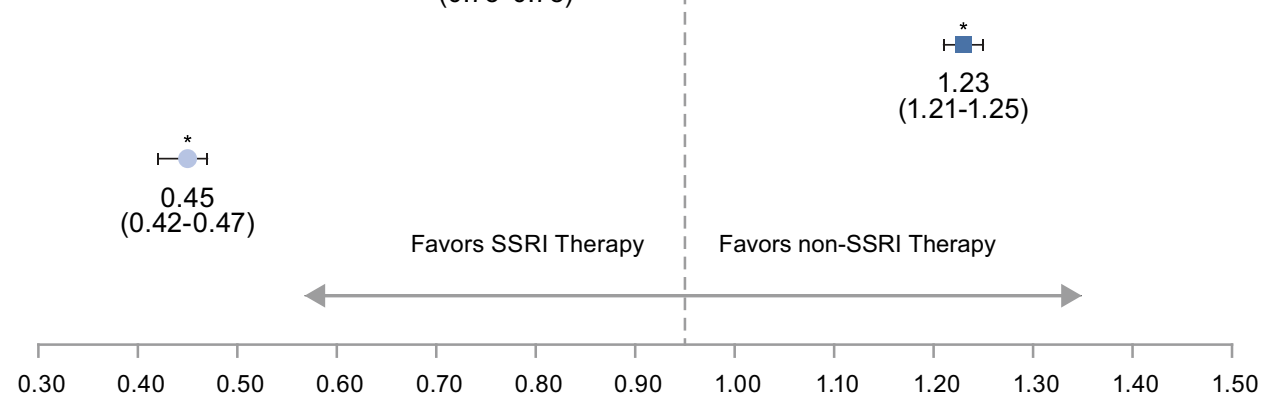

Adjusted Odds Ratio (95\% Confidence Interval)

Fig. 7 Adjusted odds of adherence at 6 months versus initial selective serotonin reuptake inhibitor therapy ${ }^{\mathrm{a}, \mathrm{b}} . * p<0.0001$ vs. initial selective serotonin reuptake inhibitor therapy. ${ }^{\text {a Results (odds }}$ ratios of adherence and 95\% confidence intervals) were similar for the 3-, 9-, and 12-month time frames. Results for monoamine oxidase inhibitors were non-significant at all timeframes and were not shown here because of small sample size $(n=85$, odds ratio $=1.00,95 \%$ confidence interval $0.64-1.56)$. ${ }^{\mathrm{b}}$ Covariates included baseline

influence patient adherence and persistence, particularly the role of the provider.

\section{Conclusion}

Based on a retrospective analysis of a large insurance claims database, we found that patients with MDD demonstrated low adherence and persistence to initial $\mathrm{AD}$ demographic covariates: age, sex, source of insurance claim, region in which medical care was received, and type of health plan. Clinical covariates were major depressive disorder diagnosis code, Charlson Comorbidity Index, presence of a comorbid anxiety disorder, and presence of a comorbid chronic non-cancer pain disorder. $A D$ antidepressant, $S N R I$ serotonin and norepinephrine reuptake inhibitor, $T C A$ tricyclic antidepressant

therapy. Adherence and persistence overall to AD therapy was only 35 and 36\%, respectively, at 6 months after initiating therapy. Our results suggest that adherence may differ by therapeutic class; patients initiating SNRI therapy appeared to have a higher likelihood of adherence than those receiving initial SSRI therapy over the year assessed. Persistence may also differ by therapeutic class, with initial SNRI therapy appearing to be associated with greater persistence than other therapeutic classes over the year 
assessed. Taking into account limitations of the insurance claims database when interpreting these results, further study is required to confirm our findings and determine additional drivers, such as pharmacological properties and other clinical variables, of these apparent differences by AD therapeutic class.

Acknowledgements Editorial assistance (eg, minor copy editing and manuscript formatting) and graphics assistance (eg, figure formatting) were provided by Jill Shults, PhD, and Bill Sterling, respectively, of Prescott Medical Communications Group (Chicago, Illinois), a contractor of Allergan.

\section{Compliance with Ethical Standards}

Funding This research was funded as part of the University of Washington/Allergan Fellowship Program through the University of Washington's Pharmaceutical Outcomes Research and Policy Program, as well as by Allergan. Open access fees were funded by Allergan.

Conflicts of interest Katelyn R. Keyloun and Patrick Gillard are fulltime employees of Allergan. Zsolt Hepp was an employee of Allergan at the time of the study. Michael E. Thase has received grants from the Agency for Healthcare Research and Quality, Alkermes, AssureRx, Avanir, Forest Laboratories (an Allergan affiliate), Intracellular, Janssen, the National Institute of Mental Health, Otsuka, PharmaNeuroboost, Roche, and Takeda; has acted as an advisor or consultant for Acadia, Alkermes, Allergan (Forest Laboratories, Naurex), AstraZeneca, Bristol-Myers Squibb, Cerecor, Eli Lilly, Fabre-Kramer Pharmaceuticals, Inc., Gerson Lehman Group, GlaxoSmithKline, Guidepoint Global, Johnson and Johnson (Janssen, Ortho-McNeil), Lundbeck, MedAvante, Merck, Moksha8, Nestle (PamLab), Neuronetics, Novartis, Otsuka, Pfizer, Shire, Sunovion, and Takeda; has received royalties from the American Psychiatric Association, Guilford Publications, Herald House, and W. W. Norton \& Company; and holds equity in MedAvante Inc. Ryan N. Hansen and Emily Beth Devine have no conflicts to report.

Open Access This article is distributed under the terms of the Creative Commons Attribution-NonCommercial 4.0 International License (http://creativecommons.org/licenses/by-nc/4.0/), which permits any noncommercial use, distribution, and reproduction in any medium, provided you give appropriate credit to the original author(s) and the source, provide a link to the Creative Commons license, and indicate if changes were made.

\section{References}

1. Andrade L, Caraveo-Anduaga JJ, Berglund P, Bijl RV, De Graaf $\mathrm{R}$, Vollebergh $\mathrm{W}$, et al. The epidemiology of major depressive episodes: results from the International Consortium of Psychiatric Epidemiology (ICPE) Surveys. Int J Methods Psychiatr Res. 2003;12(1):3-21

2. Compton WM, Conway KP, Stinson FS, Grant BF. Changes in the prevalence of major depression and comorbid substance use disorders in the United States between 1991-1992 and 2001-2002. Am J Psychiatry. 2006;163(12):2141-7.

3. Substance Abuse and Mental Health Services Administration. Results from the 2013 National Survey on Drug Use and Health: Mental Health Findings. 2014. http://www.samhsa.gov/data/sites/ default/files/NSDUHmhfr2013/NSDUHmhfr2013.pdf. Accessed 7 July 2016.
4. Greenberg PE, Kessler RC, Birnbaum HG, Leong SA, Lowe SW, Berglund PA, et al. The economic burden of depression in the United States: how did it change between 1990 and 2000? J Clin Psychiatry. 2003;64(12):1465-75.

5. American Psychiatric Association (APA). Practice guidelines for the treatment of patients with major depressive disorder, 3rd edition. Arlington, VA: APA; 2010. http://psychiatryonline.org/ guidelines. Accessed 7 July 2016.

6. Melfi CA, Chawla AJ, Croghan TW, Hanna MP, Kennedy S, Sredl K. The effects of adherence to antidepressant treatment guidelines on relapse and recurrence of depression. Arch Gen Psychiatry. 1998;55(12):1128-32.

7. Sood N, Treglia M, Obenchain RL, Dulisse B, Melfi CA, Croghan TW. Determinants of antidepressant treatment outcome. Am J Manag Care. 2000;6(12):1327-36.

8. Hirschfeld RM. Clinical importance of long-term antidepressant treatment. Br J Psychiatry Suppl. 2001;42:S4-8.

9. Akerblad AC, Bengtsson F, von Knorring L, Ekselius L. Response, remission and relapse in relation to adherence in primary care treatment of depression: a 2-year outcome study. Int Clin Psychopharmacol. 2006;21(2):117-24.

10. Cramer JA, Roy A, Burrell A, Fairchild CJ, Fuldeore MJ, Ollendorf DA, et al. Medication compliance and persistence: terminology and definitions. Value Health. 2008;11(1):44-7.

11. Katon W, Cantrell CR, Sokol MC, Chiao E, Gdovin JM. Impact of antidepressant drug adherence on comorbid medication use and resource utilization. Arch Intern Med. 2005;165(21):2497-503.

12. Cantrell CR, Eaddy MT, Shah MB, Regan TS, Sokol MC. Methods for evaluating patient adherence to antidepressant therapy: a real-world comparison of adherence and economic outcomes. Med Care. 2006;44(4):300-3.

13. Krivoy A, Balicer RD, Feldman B, Hoshen M, Zalsman G, Weizman A, et al. Adherence to antidepressants is associated with lower mortality: a 4-year population-based cohort study. J Clin Psychiatry. 2016;77(5):e566-72.

14. Sheehan DV, Keene MS, Eaddy M, Krulewicz S, Kraus JE, Carpenter DJ. Differences in medication adherence and healthcare resource utilization patterns: older versus newer antidepressant agents in patients with depression and/or anxiety disorders. CNS Drug. 2008;22(11):963-73.

15. Prukkanone B, Vos T, Burgess P, Chaiyakunapruk N, Bertram M. Adherence to antidepressant therapy for major depressive patients in a psychiatric hospital in Thailand. BMC Psychiatry. 2010;10:64.

16. Wu CH, Farley JF, Gaynes BN. The association between antidepressant dosage titration and medication adherence among patients with depression. Depress Anxiety. 2012;29(6):506-14.

17. Sawada N, Uchida H, Suzuki T, Watanabe K, Kikuchi T, Handa $\mathrm{T}$, et al. Persistence and compliance to antidepressant treatment in patients with depression: a chart review. BMC Psychiatry. 2009;9:38.

18. National Committee for Quality Assurance (NCQA). The State of Health Care Quality Report 2014. 2014. http://store.ncqa.org/ index.php/2014-state-of-health-care-quality-report.html. Account required. Accessed 7 July 2016.

19. Application: Specimen or Data Use, Non-Identifiable (and selfdetermination) version 5.70. UW Human Subjects Division. 2016. http://www.washington.edu/research/hsd/docs/295. Accessed 7 July 2016.

20. Truven Health Analytics MarketScan Database: Better Understand Health Economics and Treatment Outcomes. 2016. http:// truvenhealth.com/your-healthcare-focus/life-sciences/datadatabases-and-online-tools. Accessed 7 July 2016.

21. Marcus SC, Hassan M, Olfson M. Antidepressant switching among adherent patients treated for depression. Psychiatr Serv. 2009;60(5):617-23. 
22. Wade RL, Kindermann SL, Hou Q, Thase ME. Comparative assessment of adherence measures and resource use in SSRI/ SNRI-treated patients with depression using second-generation antipsychotics or L-methylfolate as adjunctive therapy. J Manag Care Pharm. 2014;20(1):76-85.

23. USP Medicare Model Guidelines v6.0. 2014. http://www.usp.org/ sites/default/files/usp_pdf/EN/uspmmg_v6_0_w_exampledrugs_ rev140415.pdf. Accessed 20 January 2017.

24. National Committee for Quality Assurance (NCQA). HEDIS 2015: healthcare effectiveness data and information set. 2014; 1 \& 2. http://www.ncqa.org/Portals/0/HEDISQM/Hedis2015/List_ of_HEDIS_2015_Measures.pdf. Accessed 7 July 2016.

25. Liu X, Tepper PG, Able SL. Adherence and persistence with duloxetine and hospital utilization in patients with major depressive disorder. Int Clin Psychopharmacol. 2011;26(3):173-80.

26. Wang J, Liu X, Mullins CD. Treatment adherence and persistence with duloxetine, venlafaxine XR, and escitalopram among patients with major depressive disorder and chronic pain-related diseases. Curr Med Res Opin. 2011;27(7):1303-13.

27. Qaseem A, Snow V, Denberg TD, Forciea MA, Owens DK. Clinical Efficacy Assessment Subcommittee of American College of P. Using second-generation antidepressants to treat depressive disorders: a clinical practice guideline from the American College of Physicians. Ann Intern Med. 2008;149(10):725-33.

28. Chu L-H, Kawatkar A, Gu A. A SAS ${ }^{\circledR}$ macro program to calculate medication adherence rate for single and multiple medication use. Kaiser Permanente. 2002. http://www.wuss.org/ proceedings11/Papers_Chu_L_74886.pdf. Accessed 7 July 2016.

29. Hess LM, Raebel MA, Conner DA, Malone DC. Measurement of adherence in pharmacy administrative databases: a proposal for standard definitions and preferred measures. Ann Pharmacother. 2006;40(7-8):1280-8.

30. Nau DP. Proportion of days covered (PDC) as a preferred method of measuring medication adherence. Pharm. Qual. Alliance. 2009. http://ep.yimg.com/ty/cdn/epill/pdcmpr.pdf. Accessed 7 July 2016.

31. Karve S, Cleves MA, Helm M, Hudson TJ, West DS, Martin BC. Good and poor adherence: optimal cut-point for adherence measures using administrative claims data. Curr Med Res Opin. 2009;25(9):2303-10.

32. Rost K, Dickinson LM, Fortney J, Westfall J, Hermann RC. Clinical improvement associated with conformance to HEDISbased depression care. Ment Health Serv Res. 2005;7(2):103-12.

33. Robinson RL, Long SR, Chang S, Able S, Baser O, Obenchain RL, et al. Higher costs and therapeutic factors associated with adherence to NCQA HEDIS antidepressant medication management measures: analysis of administrative claims. J Manag Care Pharm. 2006;12(1):43-54.

34. Deyo RA, Cherkin DC, Ciol MA. Adapting a clinical comorbidity index for use with ICD-9-CM administrative databases. J Clin Epidemiol. 1992;45(6):613-9.

35. Nersesyan H, Slavin KV. Current aproach to cancer pain management: availability and implications of different treatment options. Ther Clin Risk Manag. 2007;3(3):381-400.

36. Gore M, Tai KS, Sadosky A, Leslie D, Stacey BR. Clinical comorbidities, treatment patterns, and direct medical costs of patients with osteoarthritis in usual care: a retrospective claims database analysis. J Med Econ. 2011;14(4):497-507.

37. Kozma CM, Provenzano DA, Slaton TL, Patel AA, Benson CJ. Complexity of pain management among patients with nociceptive or neuropathic neck, back, or osteoarthritis diagnoses. J Manag Care Spec Pharm. 2014;20(5):455-66b.

38. StataCorp. Stata Statistical Software: Release 13. College Station, TX: StataCorp LP; 2013.

39. Takeda Pharmaceuticals. Trintellix (vortioxetine) tablets, for oral use [prescribing information]. Deerfield, IL: Takeda Pharmaceuticals; 2017. http://general.takedapharm.com/content/file. asp $\mathrm{x}$ ?filetypecode $=$ TRINTELLIXPI\&cacheRandomizer $=\mathrm{f5} \mathrm{d} 577$ cd-6fb4-4d3c-8a8d-f03843449fcb. Accessed 22 Mar 2017.

40. Forest Pharmaceuticals. Fetzima ${ }^{\circledR}$ (levomilnacipran) extendedrelease capsules, for oral use [prescribing information]. Irvine, CA: Allergan; 2014. http://www.allergan.com/assets/pdf/ fetzima_pi\#page=1. Accessed 7 July 2016.

41. Serna MC, Cruz I, Real J, Gasco E, Galvan L. Duration and adherence of antidepressant treatment (2003-2007) based on prescription database. Eur Psychiatry. 2010;25(4):206-13.

42. Wu EQ, Greenberg PE, Yang E, Yu AP, Ben-Hamadi R, Erder $\mathrm{MH}$. Treatment persistence, healthcare utilisation and costs in adult patients with major depressive disorder: a comparison between escitalopram and other SSRI/SNRIs. J Med Econ. 2009;12(2):124-35.

43. Briesacher BA, Andrade SE, Fouayzi H, Chan KA. Comparison of drug adherence rates among patients with seven different medical conditions. Pharmacotherapy. 2008;28(4):437-43.

44. Yeaw J, Benner JS, Walt JG, Sian S, Smith DB. Comparing adherence and persistence across 6 chronic medication classes. J Manag Care Pharm. 2009;15(9):728-40.

45. Stahl SM, Grady MM, Moret C, Briley M. SNRIs: their pharmacology, clinical efficacy, and tolerability in comparison with other classes of antidepressants. CNS Spectr. 2005;10(9):732-47. 